\title{
Regulation of osteoclastogenesis through Tim-3: possible involvement of the Tim-3/galectin-9 system in the modulation of inflammatory bone destruction
}

\author{
Kanako Moriyama ${ }^{1,2}$, Akiko Kukita $^{3}$, Yin-Ji Li ${ }^{1}$, Norihisa Uehara ${ }^{1}$, Jing-Qi Zhang ${ }^{1}$, Ichiro Takahashi ${ }^{2}$ and \\ Toshio Kukita ${ }^{1}$
}

\begin{abstract}
Galectins are a unique family of lectins bearing one or two carbohydrate recognition domains (CRDs) that have the ability to bind molecules with $\beta$-galactoside-containing carbohydrates. It has been shown that galectins regulate not only cell growth and differentiation but also immune responses, as well as inflammation. Galectin-9, a tandem repeat type of galectin, was originally identified as a chemotactic factor for eosinophils, and is also involved in the regulatory process of inflammation. Here, we examined the involvement of galectin- 9 and its receptor, T-cell immunoglobulin- and mucin-domain-containing molecule 3 (Tim-3), in the control of osteoclastogenesis and inflammatory bone destruction. Expression of Tim-3 was detected in osteoclasts and its mononuclear precursors in vivo and in vitro. Galectin-9 markedly inhibited osteoclastogenesis as evaluated in osteoclast precursor cell line RAW-D cells and primary bone marrow cells of mice and rats. The inhibitory effects of galectin- 9 on osteoclastogenesis was negated by the addition of $\beta$-lactose, an antagonist for galectin binding, suggesting that the inhibitory effect of galectin- 9 was mediated through CRD. When galectin-9 was injected into rats with adjuvant-induced arthritis, marked suppression of bone destruction was observed. Inflammatory bone destruction could be efficiently ameliorated by controlling the Tim-3/galectin-9 system in rheumatoid arthritis.
\end{abstract}

Laboratory Investigation (2014) 94, 1200-1211; doi:10.1038/labinvest.2014.107; published online 29 September 2014

Galectins are a family of animal lectins having the ability to regulate cell growth and differentiation, which bear at least one carbohydrate recognition domain (CRD) with high affinity for $\beta$-galactosides. Galectins are classified into three different types according to the structure: the prototype galectin with one CRD, the tandem repeat type of galectin bearing two CRDs, and the chimera type of galectin having one CRD and N-terminal peptide. ${ }^{1}$ Galectins have also been recognized as the important fine regulators of immune responses. ${ }^{2}$ Galectin-9, a tandem repeat type of galectin, was originally identified as a chemotactic factor for eosinophils 3,4 and later as a chemoattractant for neutrophils ${ }^{5}$ and NK cells. ${ }^{6}$

Inflammatory bone destruction is often associated with chronic inflammatory diseases, for example, rheumatoid arthritis and periodontal disease. Bone destruction is mediated by the professional bone-resorbing cells, osteoclasts, which are derived from hematopoietic stem cells. In rheumatoid arthritis, severe bone destruction is frequently observed in bones adjacent to arthritic joints. Joint swelling accompanied with inflammation is mainly caused by a marked increase in the thickness of the synovial membrane, in which numerous immune cells involving the helper T-cell subset; $\mathrm{T}$ helper (Th)-1 cells are recruited. ${ }^{7}$ The presence of abundant mononuclear osteoclast precursors is also present in the synovial membrane of patients with rheumatoid arthritis. ${ }^{8}$ Helper T-cell subsets are also shown to be involved in the incidence of periodontal disease, ${ }^{9}$ which could contribute to severe bone destruction in the inflammatory sites.

T-cell immunoglobulin- and mucin domain-containing molecule 3 (Tim-3) is a transmembrane protein that acts as the specific receptor for galectin-9. ${ }^{10,11}$ Tim-3 was originally discovered as the specific cell surface marker of Th1 cells, the T-cell subset inducing cellular immunity and autoimmunity. ${ }^{12}$ Immune reactions are thought to be controlled by the

\footnotetext{
Department of Molecular Cell Biology and Oral Anatomy, Faculty of Dental Sciences, Graduate School of Dental Science, Kyushu University, Fukuoka, Japan; ${ }^{2}$ Department of Orthodontics, Graduate School of Dental Science, Kyushu University, Fukuoka, Japan and ${ }^{3}$ Department of Microbiology, Faculty of Medicine, Saga University, Saga, Japan

Correspondence: Professor T Kukita, PhD, Department of Molecular Cell Biology and Oral Anatomy, Faculty of Dental Sciences, Graduate School of Dental Science, Kyushu University, 3-1-1 Maidashi, Fukuoka 812-8582, Japan.

E-mail: kukitat@dent.kyushu-u.ac.jp

Received 6 December 2013; revised 9 May 2014; accepted 19 May 2014
} 
several subsets of T cells. Recently, another T-cell subset Th17 cells have been recognized as the important subset for the progression of inflammation. ${ }^{13}$ In inflammatory sites, abundant macrophages are also recruited and work as the active forefront of innate immunity, in which activated macrophages contribute to the clearance of apoptotic cells and foreign bodies, as well as tissue repair. ${ }^{14}$ It has been reported that macrophages bearing infected parasitic bacteria produce abundant galectin- $9,{ }^{15}$ and recruited Th1 cells around the infected macrophages are stimulated by galectin- 9 through Tim- 3 to produce factors having the ability to activate macrophages, which results in killing the infected macrophages. It has been shown that Th17 cells also express Tim-3, although its expression level is lower than that in Th1 cells. ${ }^{16}$

Here we examined a possible involvement of Tim-3/galectin-9 system in the regulation of inflammatory bone destruction mediated by osteoclasts. We have obtained lines of evidence demonstrating that galectin-9 directly suppresses osteoclastogenesis through its specific receptor Tim-3 expressed on cell surface of osteoclast precursors and that the Tim-3/galectin-9 regulatory system is involved in the regulation of inflammatory bone destruction in rats with adjuvant-induced arthritis.

\section{MATERIALS AND METHODS Reagents}

Recombinant human galectin-9 was purchased from R\&D Systems (Minneapolis, MN, USA). Recombinant human galectin-9, recombinant human M-CSF, and recombinant human-soluble RANKL was from PeproTech (London, UK), and recombinant human TNF- $\alpha$ was obtained from Roche Molecular Biochemical (Mannheim, Germany). 1 $\alpha$, 25-Dihydroxy vitamin $\mathrm{D}_{3}\left(1 \alpha, 25(\mathrm{OH})_{2} \mathrm{D}_{3}\right)$ was purchased from Biomol Research Laboratories (Plymouth Meeting, UK). Leukocyte acid phosphatase kit and $\beta$-lactose were obtained from Sigma (St Louis, MO, USA). Heat-killed Mycobacterium butyricum and mineral oil were obtained from Difco Laboratories (Detroit, MI, USA). Anti-galectin-9, anti-T-cell immunoglobulin, and mucin 3 (Tim-3) were obtained from Santa Cruz Biotechnology (Santa Cruz, CA, USA). Alexa Fluor ${ }^{\circledR} 488$ Goat anti-rabbit $\operatorname{IgG}(\mathrm{H}+\mathrm{L})$ for immunofluorescence was obtained from Life Technologies Corporation (Tokyo, Japan). Biotinylated anti-goat $\operatorname{IgG}(\mathrm{H}+\mathrm{L})$ was obtained from Vector Laboratories (Burlingame, CA, USA). Peroxidase-conjugated anti-mouse and anti-rabbit IgG Abs and enhanced chemiluminescence (ECL) kits were purchased from Amersham Biosciences (Buckinghamshire, UK). Sprague-Dawley (SD) rats, Lewis rats, and C57BL/6 mice were obtained from Kyudo (Tosu, Japan). All animal experiments were performed according to the guideline for 'Care and Use of Animals of Kyushu University'.

\section{Osteoclast Differentiation}

Mouse and rat bone marrow cultures were performed as described previously. ${ }^{17-19}$ Bone marrow cells were obtained from the tibia and femur of 4-6-week-old male SD rats or 5week-old female C57BL/6 mouse. Rat bone marrow cells were cultured in 24 -well plates $\left(1 \times 10^{6}\right.$ cells per well $)$ in $\alpha$-MEM (Gibco, Grand Island, NY, USA) containing 15\% FBS (Biosource, Rockville, MD, USA) in the presence of $10^{-8} \mathrm{M}$ $1 \alpha, 25(\mathrm{OH})_{2} \mathrm{D}_{3}, 20 \mathrm{ng} / \mathrm{ml}$ of RANKL, and $10 \%$ heat-treated ROS17/2.8 cell-conditioned medium (htROSCM). Stromal cell-free bone marrow cells were prepared by passing through a Sephadex G10 column, followed by culture for 4 days in 96-multi-well plates $\left(4 \times 10^{5}\right.$ cells per well $)$ in $\alpha$-MEM (Gibco) containing 15\% FBS (Biosource, Rockville, MD, USA) in the presence of $10^{-8} \mathrm{M} 1 \alpha, 25(\mathrm{OH})_{2} \mathrm{D}_{3}, 20 \mathrm{ng} / \mathrm{ml}$ of RANKL, and 10\% htROSCM. Mouse bone marrow cells were treated with M-CSF $(20 \mathrm{ng} / \mathrm{ml})$ for 2 days, followed by culture for 3 days in the presence of M-CSF $(20 \mathrm{ng} / \mathrm{ml})$ and RANKL $(50 \mathrm{ng} / \mathrm{ml})$. The osteoclast precursor cell line, RAW$\mathrm{D}$, is a subclone of murine macrophage cell line, RAW264, and has an extremely high potential ability to differentiate into osteoclasts as described previously. ${ }^{20,21}$ RAW-D cells were cultured in 96 -well plates $\left(6.8 \times 10^{3}\right.$ cells per well $)$ in $\alpha$-MEM containing 10\% FBS for 3 days in the presence of RANKL $(20 \mathrm{ng} / \mathrm{ml})$ and TNF- $\alpha(1 \mathrm{ng} / \mathrm{ml})$. For estimating osteoclastogenesis, cells were fixed and stained for tartrate-resistant acid phosphatase (TRAP) by use of the leukocyte acid phosphatase kits (Sigma, St Louis, MO, USA). The number of TRAP-positive multinucleated cells containing three or more nuclei was counted under a microscope. MTT assay was performed by use of PrestoBlue Cell Viability Reagent (Invitrogen). Absorbance was read at $570 \mathrm{~nm}$.

\section{Induction of Adjuvant Arthritis in Rats and Micro-CT Analysis}

Adjuvant arthritis was induced as described previously. ${ }^{22}$ Five-week-old female Lewis rats were anesthetized with diethyl ether and were intradermally injected at the base of the tail with complete Freund adjuvant (CFA) containing $25 \mathrm{mg} / \mathrm{kg}$ heat-killed M. butyricum (Difco Laboratories) suspended in mineral oil. For the control experiments, rat were injected with mineral oil alone. Galectin-9 was injected into the areas of right ankle joints from the base of the foot pad by use of $30 \mathrm{G}$ needles. Vehicle control PBS was also injected into the corresponding area of the left ankle joints of the same AA rats. Micro-CT analysis was performed mainly as described previously. ${ }^{23}$ The animals were killed and fixed in $4 \%$ paraformaldehyde at the end of the experiments, and the hind paws were collected and performed radiographic analysis using the micro-CT Skyscan 1076 scanner (Skyscan, Konitich, Belgium). Strength of the radiation in scanning was set to $80 \mathrm{kV}$ and $200 \mu \mathrm{A}$, and the section thickness was $9 \mu \mathrm{m}$ for one scan image. Clinical scores were determined using a visual scoring method, which was defined as follows: grade 0 , no swelling; grade 1, detectable swelling in a single digit; grade 2, swelling in more than one digit; grade 3 , swelling of all digits and the instep; and grade 4 , severe swelling of the paw and ankle. Each limb was graded, yielding a maximum possible score of 16 per animal. 


\section{Immunofluorescence Staining}

After anesthetizing with isoflurane or ethylether, rats were fixed by perfusion with $4 \%$ paraformaldehyde/PB ( $\mathrm{pH} 7.4$ ). Ankle joints were excised from the hind paws and immersed in the same fixative overnight at $4{ }^{\circ} \mathrm{C}$, followed by washing in PBS overnight at $4{ }^{\circ} \mathrm{C}$. After decalcification in $10 \%(\mathrm{w} / \mathrm{v})$ ethylene diamine tetraacetate trisodium salts (EDTA-Na3) for 3 weeks at $4{ }^{\circ} \mathrm{C}$, the tissue blocks were embedded in OCT compounds. Frozen sections $(10 \mu \mathrm{m})$ of ankle joints involving tibia-tarsal-calcaneus bones were prepared by use of the cryostat (Thermo Scientific ${ }^{\mathrm{TM}}$ HM 560, USA) and immunofluorescence staining was performed. After blocking the nonspecific binding sites with $10 \%$ goat serum for $2 \mathrm{~h}$ at room temperature, sections were incubated with anti-Tim-3 rabbit polyclonal antibody raised against amino acids 111281 mapping at the $\mathrm{C}$ terminus of Tim-3 mouse origin (1:200 dilution) in a humidified chamber overnight at $4{ }^{\circ} \mathrm{C}$. After washing with $\mathrm{PBS}$, sections were incubated with goat antirabbit $\operatorname{IgG}(\mathrm{H}+\mathrm{L})$ conjugated with Alexa Fluor ${ }^{\circledR} 488$ (1:500 dilution; Molecular Probes, Life Technologies Inc., Carlsbad, CA, USA) for $1 \mathrm{~h}$ at room temperature. Microscopic observations were performed by use of the fluorescence microscope (BZ-8100; Keyence, Osaka, Japan). Sections were also stained for TRAP after observation with fluorescence microscope.

\section{Immunohistochemical Staining}

After blocking the nonspecific binding sites with $10 \%$ normal rabbit serum for $1 \mathrm{~h}$ at room temperature, frozen sections $(10 \mu \mathrm{m})$ were prepared and incubated with anti-galectin-9 goat polyclonal antibody raised against a peptide mapping near the C terminus of galectin-9 of human origin (1:200 dilution) in a humidified chamber for $1 \mathrm{~h}$ at room temperature. After washing with PBS, sections were incubated with biotinylated anti-goat IgG $(\mathrm{H}+\mathrm{L})$ (rabbit IgG) (1:500 dilution; Vector Laboratories, Burlingame, CA, USA) for $30 \mathrm{~min}$ at room temperature. After washing with PBS, Vectastain ABC-AP Kit (Vector Laboratories) for $30 \mathrm{~min}$ at room temperature. Sections were treated with $0.1 \%(\mathrm{w} / \mathrm{v})$ Levamisole in $0.1 \mathrm{M}$ Tris- $\mathrm{HCl}(\mathrm{pH} 8.3)$, and color development was performed in the presence of $0.1 \%(\mathrm{w} / \mathrm{v})$ levamisole using alkaline phosphatase substrate kitl (Vector Laboratories). Adjacent sections were also stained for TRAP.

\section{RT-PCR and Real-Time PCR}

Total RNA was extracted by use of ISOGEN (Nippon Gene, Tokyo, Japan). Frozen bone tissue powders were prepared in the liquid nitrogen from the ankle joints to prepare total RNAs. Total RNAs were also prepared from cultured cells and subjected to PCR using RT-PCR Kit (Takara Bio, Otsu, Japan) according to the manufacturer's protocol. After synthesizing cDNA from the total RNA using oligo-dT primers, PCR reactions were performed using the following primers: mouse Tim-3 forward, $5^{\prime}$-ATTCTGTCCTTGGTCA CAGTGTACC-3'; mouse Tim-3 reverse, 5'-TGGTTAGGGTT
CTTGGAGAAGCTG- ${ }^{\prime}$; rat GAPDH forward, $5^{\prime}$-CCCAATG TATCCGTTGTGGATCTG-3'; rat GAPDH reverse, 5'-GTGG TCCAGGGTTTCTTACTCCTT- $3^{\prime}$. PCR products were amplified using the following parameters: $94{ }^{\circ} \mathrm{C}$ for $30 \mathrm{~s}, 60^{\circ} \mathrm{C}$ for $30 \mathrm{~s}$, and $68^{\circ} \mathrm{C}$ for $1 \mathrm{~min}$. PCR products were subjected to electrophoresis on $2 \%$ agarose gels and visualized by ethidium bromide staining with UV light illumination.

For quantitative RT-PCR, total RNA was reverse transcribed with a PrimeScript RT-PCR Kit (Takara Bio) by use of random primers. Real-time PCR reactions were performed by using SYBR Premix Ex Taq (Takara Bio) with a Step One Plus Real-Time PCR System (Applied Biosystems). mRNA levels were normalized to GAPDH expression. Commercially available probe-primer sets (Applied Biosystems) with proprietary sequences were used. Primer sequences used were as follows: Tim-3 5'-primer: AGCTAAAGGGCGATCTCAAC; Tim-3 3'-primer: CTCCGTGGTTAGGGTTCTTG; GAPDH 5'-primer: TGCACCACCAACTGCTTAG; GAPDH 3'-primer: GGATGCAGGGATGATGTTC.

\section{Western Blotting}

Cell lysates were prepared as described previously. ${ }^{22}$ The total proteins were extracted in the lysis buffer containing $1 \%$ Triton X-100, 0.5\% NP-40, $150 \mathrm{mM} \mathrm{NaCl}, 50 \mathrm{mM}$ Tris- $\mathrm{HCl}$ (pH 7.4), $1 \mathrm{mM} \mathrm{NaF}, 1 \mathrm{mM} \mathrm{Na}_{3} \mathrm{VO}_{4}, 1 \mathrm{mM}$ phenylmethylsulfonyl fluoride, and protease inhibitor cocktail (Sigma, St Louis, MO, USA). Cell lysates, containing equal amounts of proteins, were subjected to $10 \%$ SDS-PAGE and transferred to nitrocellulose membranes. After blocking with 5\% $(\mathrm{w} / \mathrm{v})$ non-fat dry milk in TBS, the membranes were probed with anti-Tim-3 antibody for $1 \mathrm{~h}$. After rinsing in TBS-Tween, the membranes were incubated with peroxidase-conjugated donkey anti-goat IgG antibody for $1 \mathrm{~h}$. The immunoreacted bands were visualized by ECL (Amersham). The same membranes were reprobed with anti-GAPDH antibody (Amersham, GE-Healthcare, Pittsburgh, PA, USA). The same membranes were reprobed with anti-GAPDH.

\section{Statistical Analysis}

All data are represented as mean \pm s.d. Statistical analysis was performed by use of Student's $t$-test.

\section{RESULTS \\ Detection of Tim-3-Positive Cells in the Inflammatory Bone Destruction Sites in Arthritic Rats}

Tim-3 was first described as a molecule specifically expressed on the cell surface of some T-cell subsets. To investigate the presence of Tim-3-positive helper $\mathrm{T}$ cells in the pathologic bone sites of arthritic rats, we attempted to find Tim-3positive cells immunohistologically in tissue sections of ankle joints. Severe bone destruction and marked elevation of osteoclastogenesis were apparent in the distal tibia of arthritic rats (Figure 1a, right panels), whereas no histologic abnormality was observed in control rats (Figure 1a, left panels). The immunofluorescence staining of the ankle joint tissues 
a
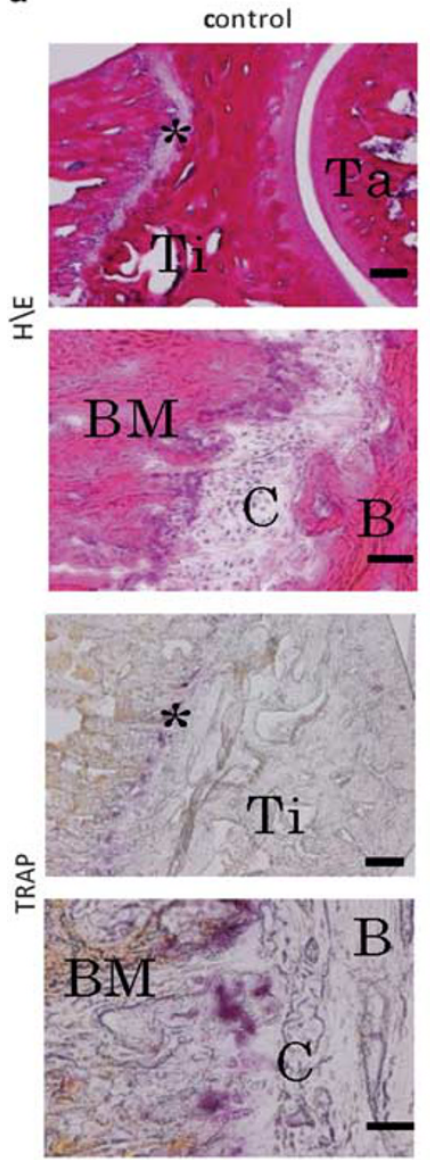

CFA
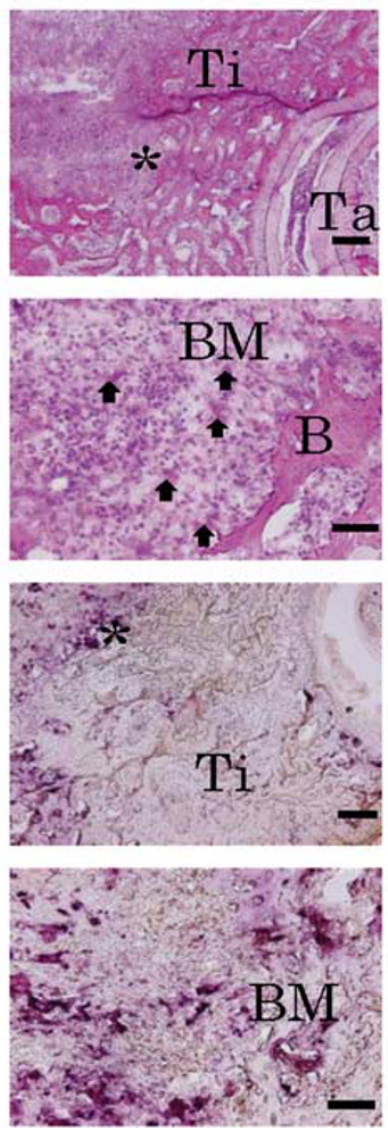

b
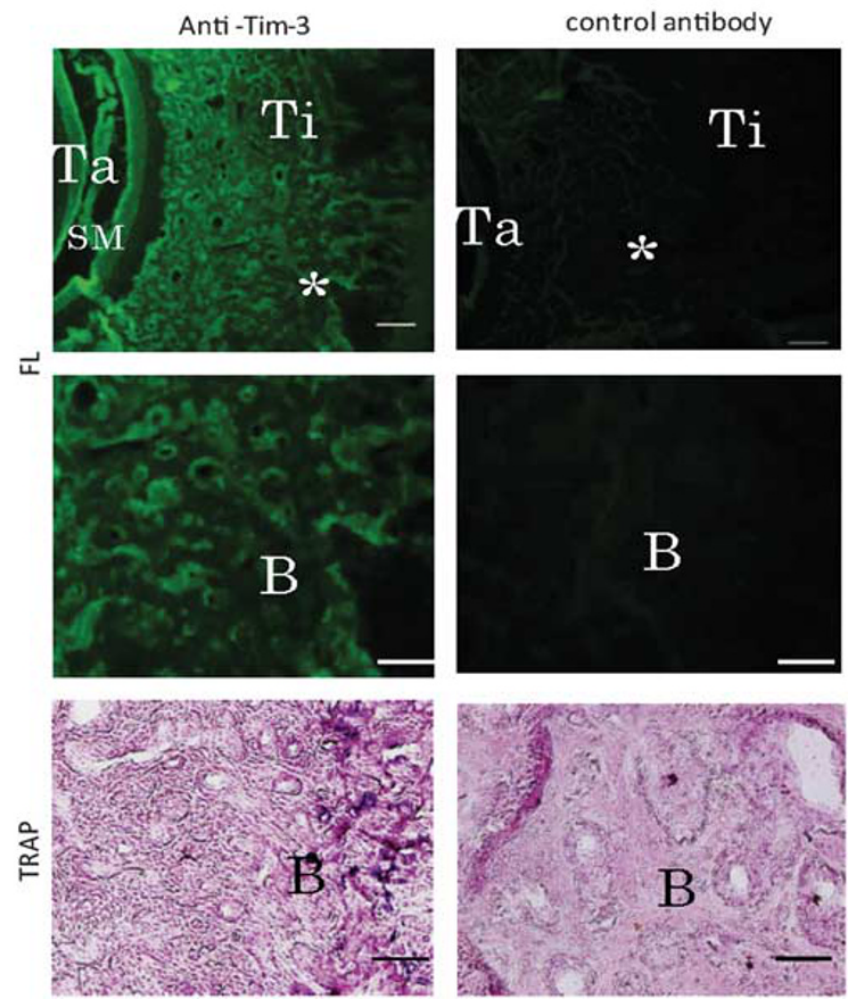

Figure 1 Induction of adjuvant-induced arthritis in rats and expression of T-cell immunoglobulin- and mucin-domain-containing molecule 3 (Tim-3) in inflammatory bone destruction sites. (a) Induction of adjuvant-induced arthritis in rats. Arthritic rats were induced by injection of complete Freund adjuvant (CFA) (right panels; CFA) as described in Materials and methods. As the vehicle control, only mineral oil was injected (left panels; control). After 21 days of CFA injection, tissue sections were prepared as described in Materials and methods. Hematoxylin/eosin staining of the ankle joints (upper four panels; HE). Lower panels show the higher magnification views of the single asterisk areas in the upper panels. Tartrate-resistant acid phosphatase (TRAP) staining of the ankle joints (lower four panels). Lower panels show the higher magnification views of the single asterisk areas in the upper panels. Bars: $100 \mu \mathrm{m}$ for the upper panels and $50 \mu \mathrm{m}$ for the lower panels. (b) Immunofluorescence (FL) analysis by use of anti-Tim-3 antibody. Ankle joint sections of arthritic rats were prepared after 21 days of injection. Cryosections of ankle joints were incubated with anti-Tim-3 polyclonal antibody followed by detection with anti-rabbit immunoglobulin G (IgG) conjugated with Alexa Fluor 488 . Bars: $200 \mu \mathrm{m}$. Middle panels show the higher magnification views of the single asterisk areas in the upper panels. Lower panels show the TRAP-staining data of the same field as to middle panels. Bars, $50 \mu \mathrm{m}$. B, bone; BM, bone marrow; C, cartilage; SM, synovial membrane; Ta, Talus; Ti, tibia.

using a anti-Tim-3 antibody demonstrated that numerous Tim-3-positive cells were present in the broad bone marrow areas among bone trabeculae of the distal tibia in arthritic rats (Figure 1b), suggesting the presence of numerous helper $\mathrm{T}$ cells in the marrow cavity of arthritic rats. Tim-3 immunoreactivity was also apparent in the synovial membranes (Figure 1b, upper panels), indicating the recruitment of Tim3 -positive $\mathrm{T}$ helper cells in the synovial tissues in arthritic rats.

\section{Expression of Tim-3 in Osteoclasts and Osteoclast Precursors In Vivo and In Vitro in Normal Conditions}

When the expression of Tim-3 in normal bone tissues of the control rats was examined (Figure $2 \mathrm{a}$ ), more restricted areas tended to be intensively stained with anti-Tim-3 antibody in comparison to Tim-3 staining data of the distal tibia of arthritic rats (Figure 1b). Tim-3-stained areas roughly corresponded to the bone surface. Tim-3-expressing cells present in the bone surface generally corresponded to TRAP-positive cells (Figure 2a, middle and lower panels of the left panels). These data suggest that osteoclasts express Tim-3 in rats with no inflammation. In contrast to the intense Tim-3 expression in synovial membranes of arthritic rats, Tim-3 immunoreactivity was not detected in normal synovial membrane.

To confirm the expression of Tim-3 in osteoclasts, immunocytochemical detection of Tim-3 protein was performed by use of cultures that form osteoclasts. RAW-D cells at 2 days of culture showed significant Tim-3 expression not only in mononuclear osteoclast precursors but also in 

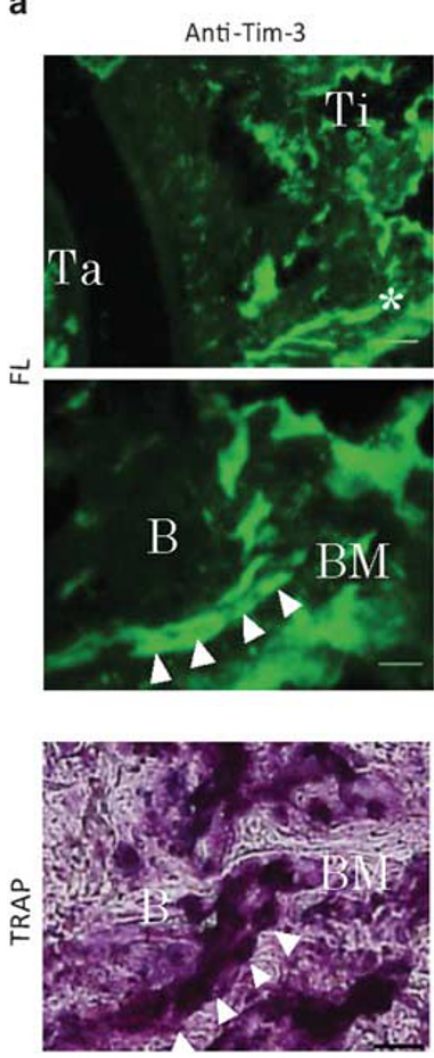

control antibody
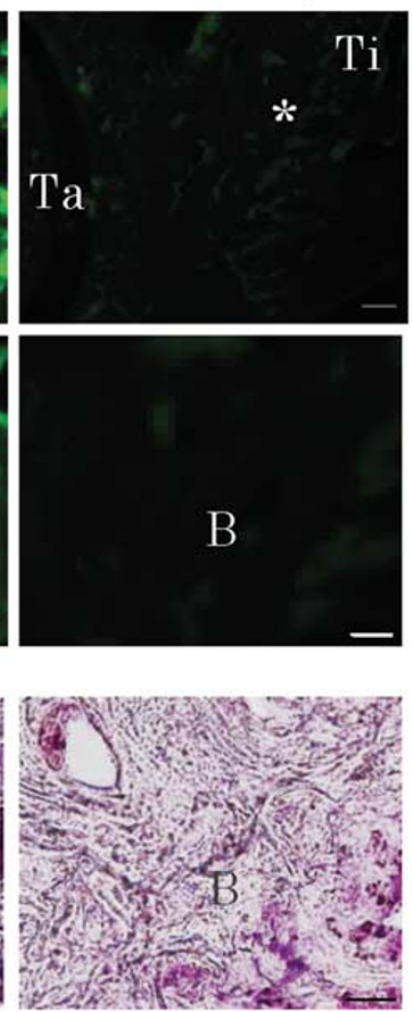

b

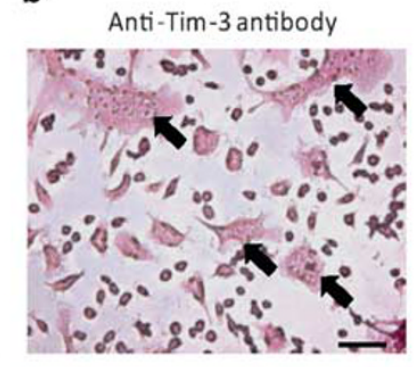

C

RAW-D

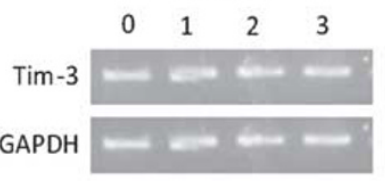

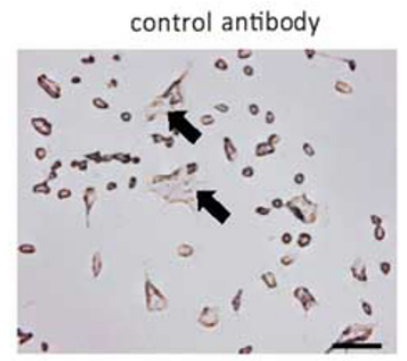

Mouse BMMs

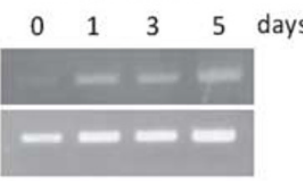

d
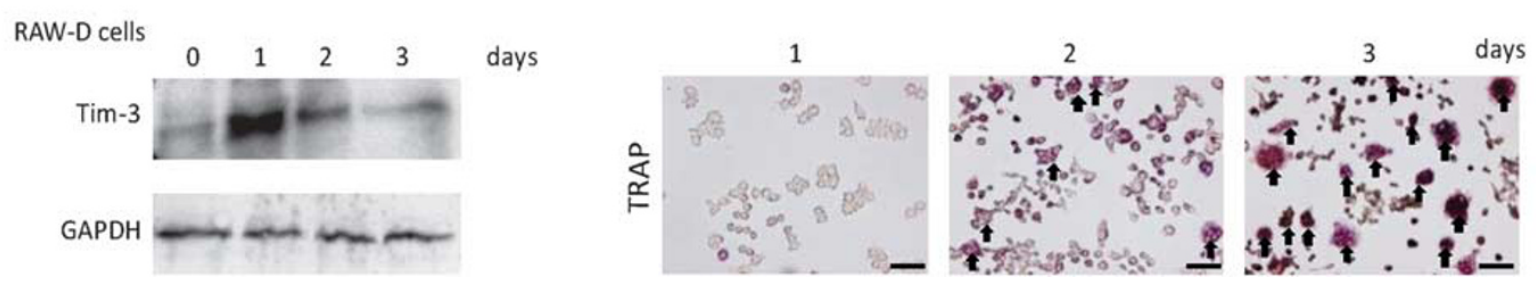

Figure 2 Expression of T-cell immunoglobulin- and mucin-domain-containing molecule 3 (Tim-3) in osteoclast precursors and osteoclasts.

(a) Immunohistochemical analysis. Ankle joints section of control rats. Cryosections of the control rats were prepared and stained with anti-Tim-3 antibody (left panels; FL) followed by detection with anti-rabbit immunoglobulin G (IgG) conjugated with Alexa Fluor 488. Adjacent sections were stained with control antibody as the primary antibody (right panels; FL). Bars, $200 \mu \mathrm{m}$. Middle panels show the higher magnification views of the single asterisk areas in upper panels. Lower panels show the histochemical TRAP-staining of the same sections of the middle panels (Anti-Tim-3 and control antibody, respectively). Arrowheads: osteoclasts. Bars: $50 \mu \mathrm{m}$. (b) Immunocytochemical detection of Tim-3 in osteoclast-like cells formed in vitro. RAW-D cells was cultured in $60 \mathrm{~mm}$ dish in the presence of receptor activator of nuclear factor kappa-B ligand (RANKL) (20 ng/ml) and tumor necrosis factor- $\alpha$ (TNF- $\alpha)(1 \mathrm{ng} / \mathrm{ml})$ for 2 days followed by fixation and immunostaining with anti-Tim-3 antibody as described in Materials and methods. Multinucleated osteoclast-like cells (MNCs) stained with anti-Tim-3 antibody (left panel). Cells were also stained with control antibody (right panel). Bars: $40 \mu$ m. Arrows: Multinucleated MNCs. (c) Gene expression of Tim-3 mRNA during osteoclastogenesis analyzed in culture of RAW-D cells and mouse bone marrow macrophages (BMMs). Semiquantitative reverse transcription-polymerase chain reaction (RT-PCR). RAW-D cells or mouse bone marrow macrophages (BMMs) were stimulated to form osteoclasts as described in Materials and methods. Total RNA was extracted and semiquantitative RT-PCR was performed. (d) Western blot analysis of Tim-3 protein expression in RAW-D cells stimulated to form osteoclasts. Total cell lysates were prepared at each time point (days in culture) and subjected to western blot analysis with indicated antibodies. Photographs show tartrate-resistant acid phosphatase (TRAP) staining of the culture at each time point. Arrows: TRAP-positive MNCs. Bars, $40 \mu \mathrm{m}$. B, bone; BM, bone marrow; Ta, Talus; Ti, tibia.

multinucleated osteoclasts, as shown in Figure 2b. Expression of Tim-3 mRNA was examined by use of semiquantitative RT-PCR (Figure 2c). In RAW-D cells, the expression of Tim-3 mRNA was detected at day 0 of culture (before stimulation) and the expression was slightly augmented at day 1 of culture. In mouse bone marrow macrophage stimulated by RANKL and M-CSF, a significant induction of Tim-3 mRNA was detected 1 day after RANKL stimulation. Real-time PCR analysis also showed stimulation of Tim-3 gene expression both in the RAW-D cell culture system and mouse bone marrow macrophage culture system (Supplementary Figure 1). Western blotting analysis of RAW-D cell 
cultures clearly showed high expression of Tim-3 protein at 1 day of culture (Figure 2d). These data demonstrate the expression of Tim-3 in cells in osteoclast lineage, osteoclast precursors, and osteoclasts.

\section{Expression of Galectin-9 In Vivo}

To understand the role of galectin-9 in the regulation of inflammatory bone destruction, we examined its expression in the distal tibia of arthritic rats with severe bone destruction around the ankle joints by use of immunohistochemical analysis. As shown in Figure 3, galectin-9 immunoreactivity was observed almost in the whole area of the bone marrow cavity (Figure 3, right panels); in contrast, galectin-9 immunoreactivity was observed in the narrow peripheral zone of the bone marrow facing to bone trabecule in control rats (Figure 3, left panels). These observations suggest that galectin-9 is actually present in the bone marrow cavity in vivo.

\section{Inhibition of Osteoclastogenesis by Galectin-9 In Vitro}

To estimate the role of galectin-9 observed in vivo, we have examined the effect of galectin- 9 on osteoclastogenesis by use of an in vitro culture system for evaluating osteoclast differentiation. Recombinant human galectin-9 markedly suppressed osteoclastogenesis in a dose-dependent manner in four different culture systems used for evaluating osteoclastogenesis. Galectin-9 significantly inhibited formation of TRAP-positive multinucleated osteoclasts in the culture system using osteoclast precursor line RAW-D cells (Figures 4a and b). Marked inhibition of osteoclastogenesis was also observed in primary culture system using bone marrow macrophages of mice (Figure 4c). Similar inhibition of osteoclastogenesis was observed in rat whole bone marrow cultures (Figure 4d). In rat bone marrow cells depleted of stromal cells, osteoclastogenesis was significantly suppressed, demonstrating a direct action of galectin-9 on cells in osteoclast lineage (Figure 4e). These data show that galectin-9
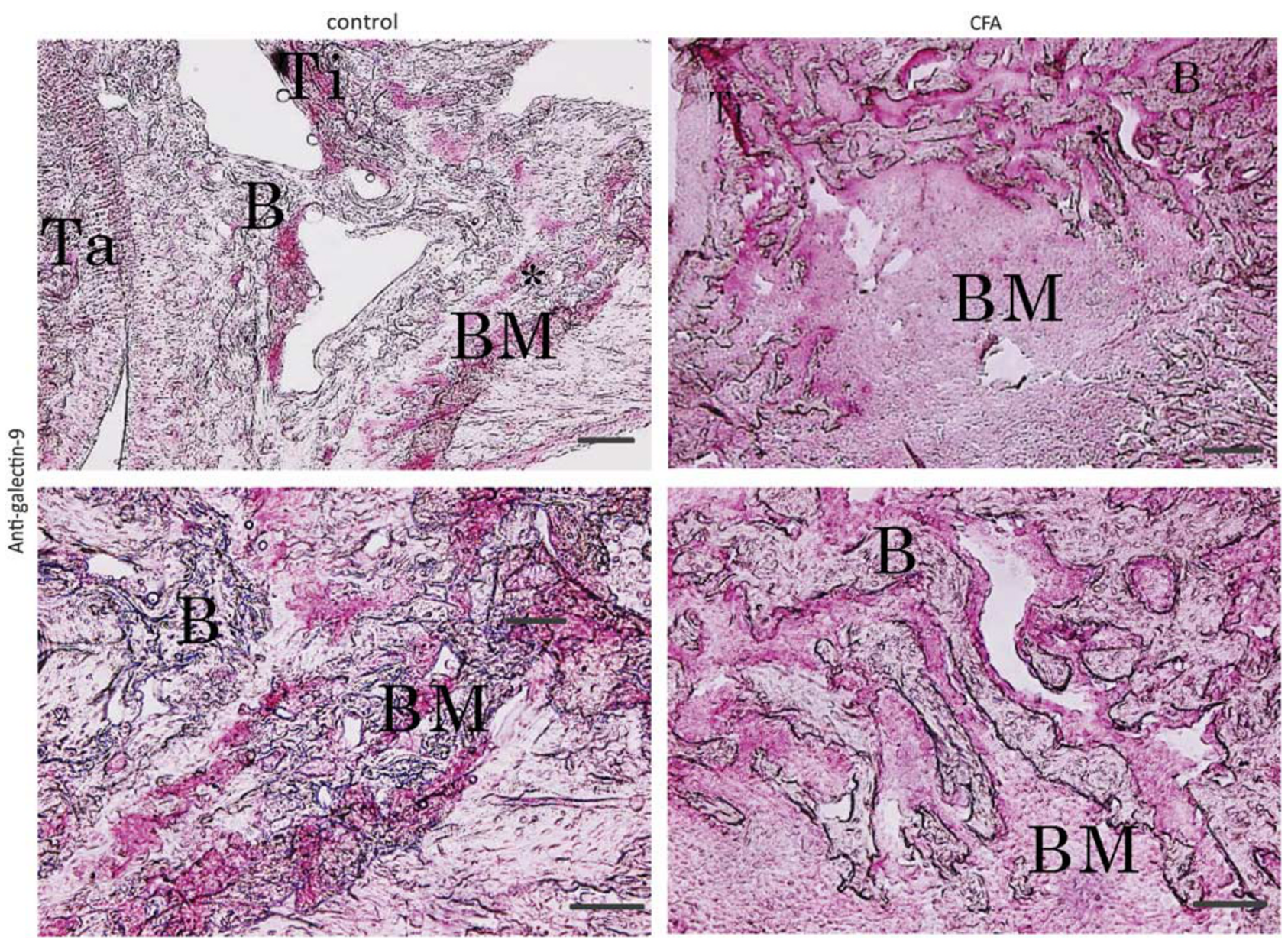

Figure 3 Immunohistochemical detection of galectin-9 in distal tibia of arthritic rats with severe bone destruction. Cryosection of the ankle joints prepared from control rats (left panels: control) and arthritic rats (right panels: complete Freund adjuvant (CFA)) were stained with aniti-galectin-9 antibody followed by detection with ABC-AP kit as described in Materials and methods. Control: injected only with Mineral oil (left panels). CFA: Rats injected with CFA to form arthritic rats (right panels). Lower panels demonstrate high-magnification views of the area with asterisk in the upper panels. Bars: $200 \mu \mathrm{m}$ for the upper panels and $50 \mu \mathrm{m}$ for the lower panels. B, bone; BM, bone marrow; Ta, talus; Ti, tibia. 
a

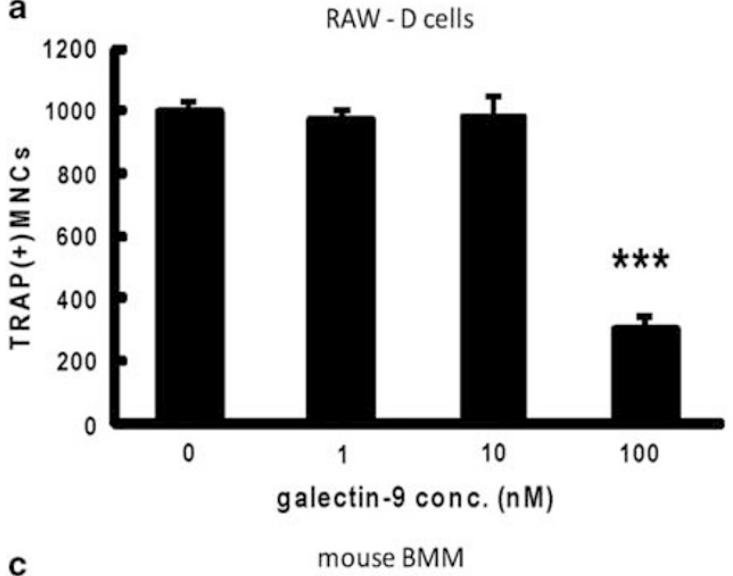

C

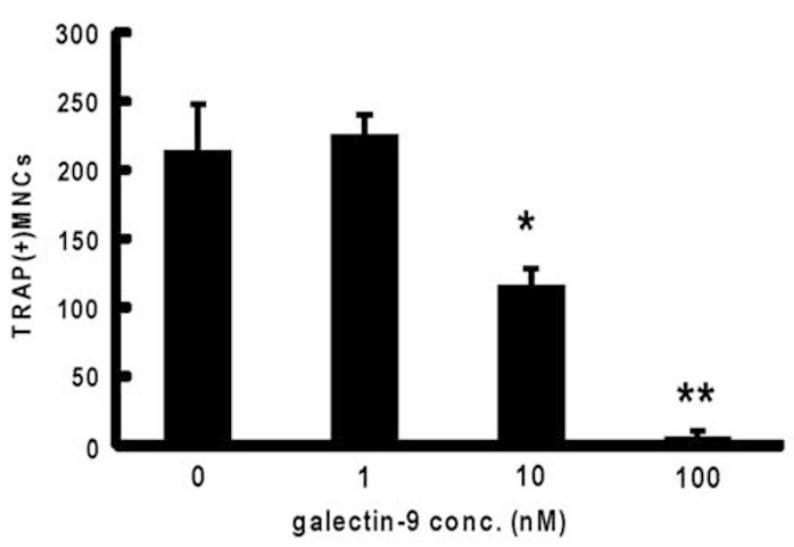

b

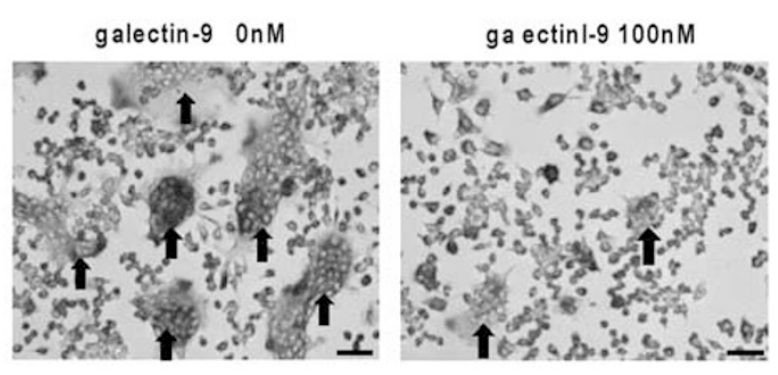

d

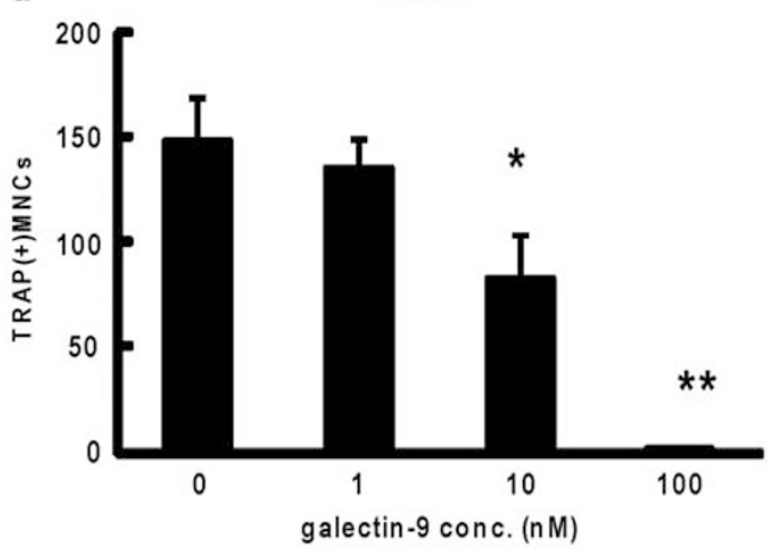

e

Stromal cell - free rat BM

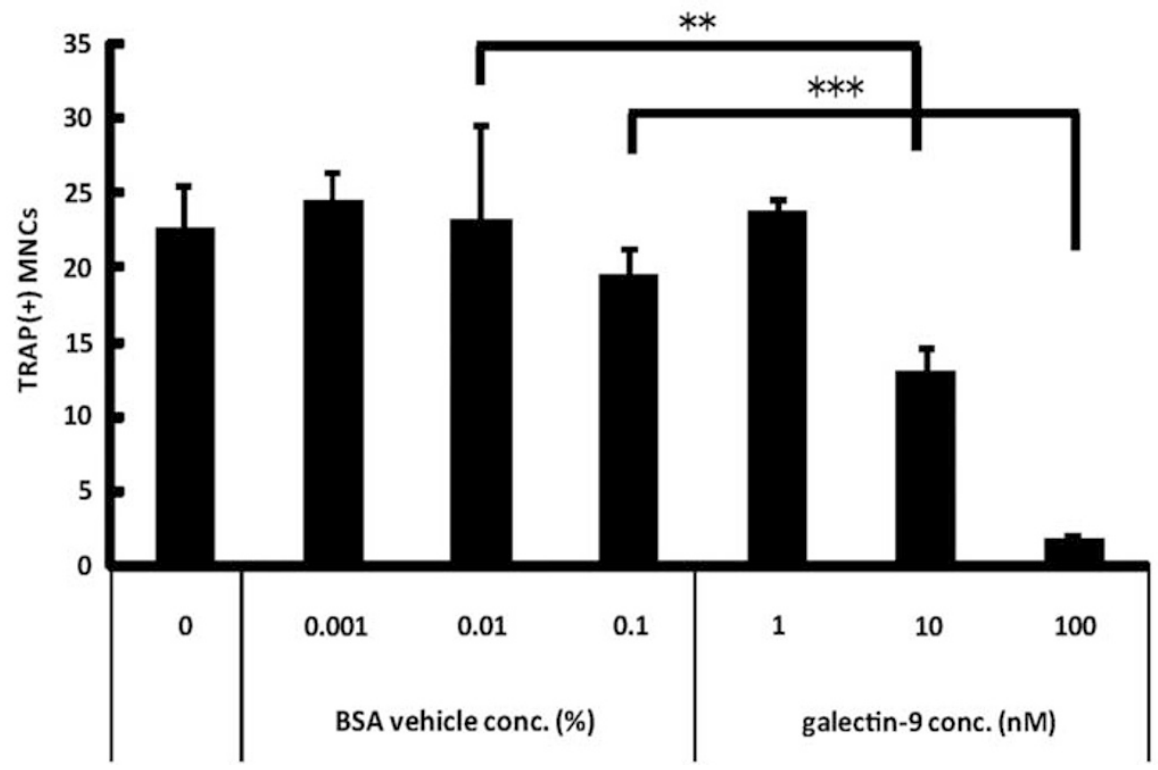

Figure 4 Inhibition of osteoclastogenesis by galectin-9. Human recombinant galectin-9 was added to each culture system evaluating osteoclastogenesis as described in Materials and methods. Cells were fixed and stained for tartrate-resistant acid phosphatase (TRAP), and the number of TRAPpositive multinucleated cells (MNCs) was counted. (a and b) RAW-D cell culture system. Arrows show TRAP-positive MNCs. Bars: $20 \mu \mathrm{m}$. (c) Mouse bone marrow macrophage system. (d) Rat whole bone marrow culture system. (e) Stromal cell-free rat bone marrow cells stimulated with receptor activator of nuclear factor kappa-B ligand (RANKL). Data were analyzed by Student's $t$-test. ${ }^{*} P<0.05,{ }^{* *} P<0.01,{ }^{* * *} P<0.001$ compared with the culture without galectin-9. 
is an effective and potent inhibitor of osteoclastogenesis in vitro. As shown in Supplementary Table 1, we confirmed that galectin-9 treatment did not reduce cell viability estimated by using MTT assay despite inhibiting osteoclasogenesis in RAW-D cell culture system of Figures $4 \mathrm{a}$ and $\mathrm{b}$.

\section{Elimination of Galectin-9-Mediated Inhibition of Osteoclastogenesis by $\beta$-Lactose}

As the function of galectins are generally mediated through $\mathrm{CRD}$, biologic activities of galectins on cell signaling are reversed by the presence of the antagonists for galectin binding, galactose or $\beta$-lactose, a disaccharide consisting of galactose and glucose. To determine whether the inhibitory effect of recombinant galectin-9 on osteoclastogenesis is mediated through CRD of galectin-9, the RAW-D cell cultures were treated with galactose or $\beta$-lactose. Although the inhibitory effects of galectin-9 on osteoclastogenesis was not reversed by the addition of galactose (Figure 5a), they were eliminated by the addition of $\beta$-lactose (Figure $5 \mathrm{~b}$ and $5 \mathrm{c}$ ). These data strongly suggest that the inhibitory effect of galectin-9 on osteoclastogenesis was mediated through CRD of galectin-9.

\section{Inhibition of Inflammatory Bone Destruction by Galectin-9 in Arthritic Rats}

To define the response of the Tim-3/galectin-9 system in vivo after the addition of a large amount of exogenously administered galectin-9, we examined whether injected galectin-9 affects bone destruction in arthritic rats. Each arthritic rat was given injections around the right or left ankle joint with recombinant galectin-9 or vehicle control PBS, respectively. Galectin-9 slightly suppressed joint inflammation; however, its suppression did not reach to a statistical significance when clinical scores were estimated (Figure 6). Figure 7 shows radiograms of the same ankle joints as those shown in Figure 6a, in which only a slight suppression of inflammation was observed by the injection of galectin- 9 . Injection of CFA induced aggressive bone destruction (Figure 7, upper right panel; CFA). A marked bone destruction was also observed when the vehicle control (CFA/PBS) was injected to the left ankle of the arthritic rat (Figure 7, lower left panel; $\mathrm{CFA} / \mathrm{PBS}$ ). Severe bone destruction was observed especially in tarsus as well as in the distal tibia (CFA, CFA/PBS). In contrast, galectin-9 injection at the right ankle of the arthritic rat markedly suppressed bone destruction in distal tibia and tarsus (CFA/galectin-9). A significant inhibition of bone destruction was apparent, especially in tarsus. These data strongly suggest that recombinant galectin-9 suppressed inflammatory bone destruction in arthritic rats.

\section{DISCUSSION}

In the current study, we elucidated that osteoclasts and osteoclast precursors expressed Tim-3 and that osteoclast differentiation was negatively regulated by its ligand galectin- 9 . In rats with adjuvant-induced arthritis, an animal model of a

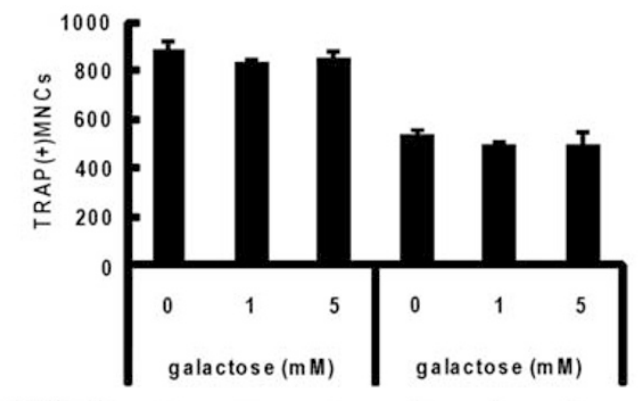

galectin- 9

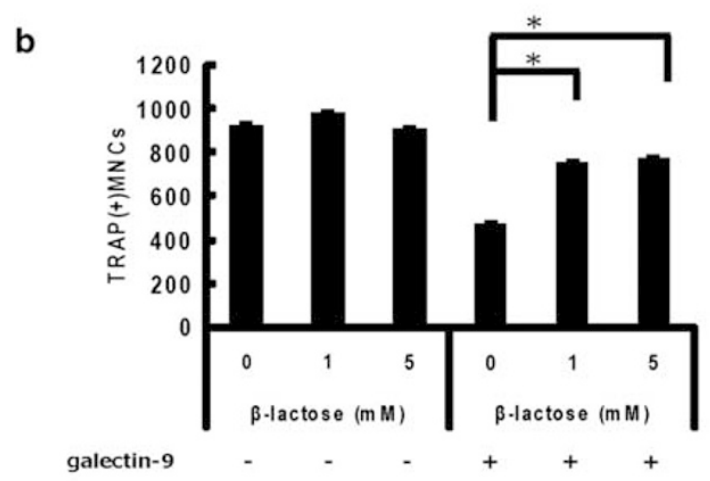

C

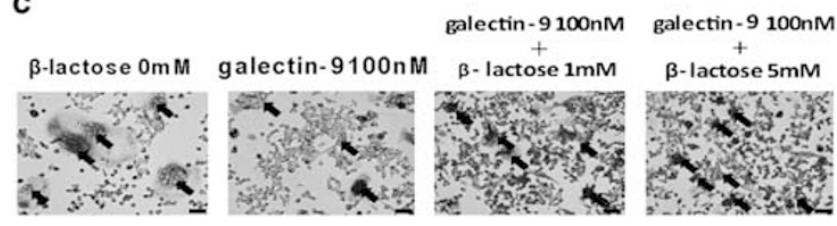

Figure 5 Cancellation of galectin-9-mediated inhibition of osteoclastogenesis by $\beta$-lactose. RAW-D cells were stimulated to form osteoclasts with or without galectin-9 $(100 \mathrm{nM})$ in the presence of galactose (a) or $\beta$-lactose (b). (c) Photographs of tartrate-resistant acid phosphatase (TRAP)-stained cultures at 3 days. Cells were stained for TRAP at 3 days and the number of multinucleated cells (MNCs) was counted. Arrows: TRAP-positive MNCs (c). Data represent a typical data from three independent experiments. Data were analyzed by Student's $t$-test compared with cultures without galactose or $\beta$-lactose. ${ }^{*} P<0.05$. Bars: $20 \mu \mathrm{m}$.

human rheumatoid arthritis, we have successfully shown a marked suppression of inflammatory bone destruction by galectin-9. Previous reports have shown that galectin-9 is expressed in synovial tissues and fibroblast-like synoviocytes obtained from patients with rheumatoid arthritis. ${ }^{24}$ When arthritic bone destruction occurs, it is believed that osteoclast precursors as well as inflammatory cells accumulated in the inflamed synovial membrane and migrate and invade into the sites of future bone destruction. ${ }^{25}$ Galectin-9 expression in the synovial tissues was also reported in mouse collageninduced arthritis (CIA) model. ${ }^{26}$ Recently, it has been shown 
a

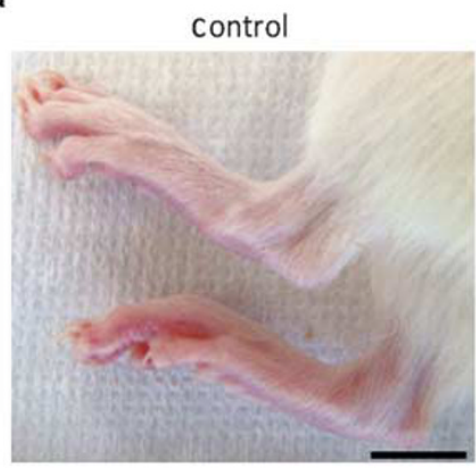

b

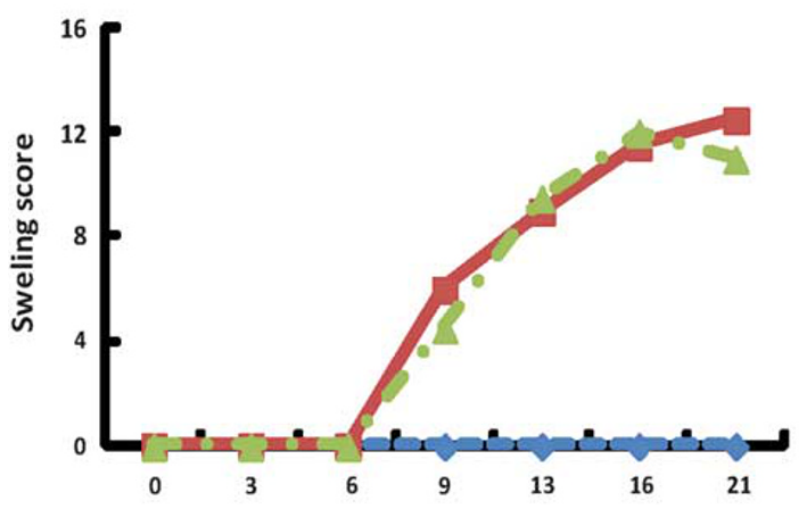

d

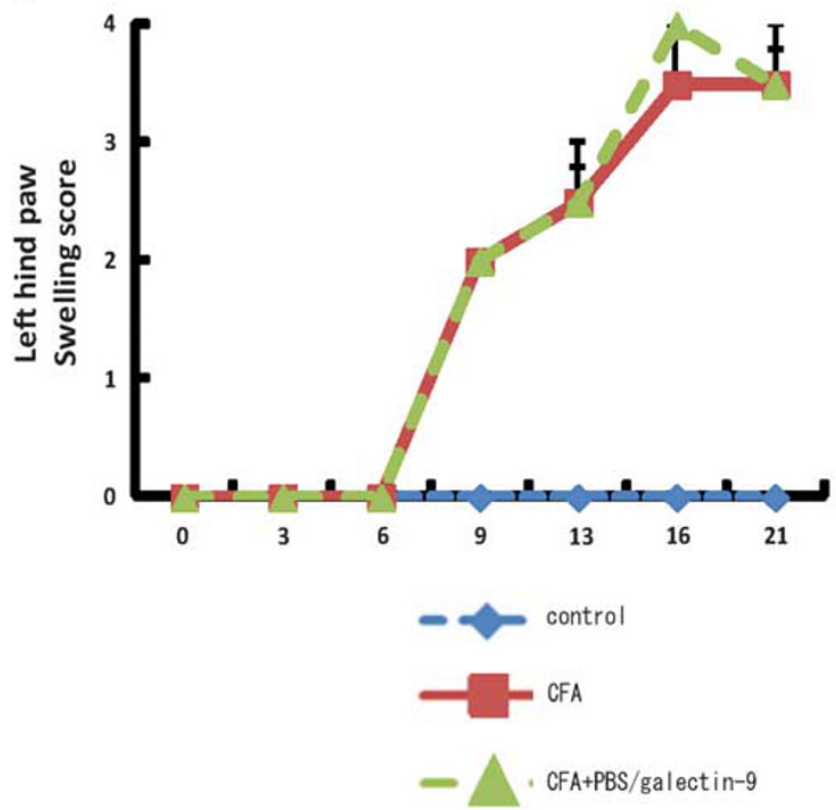

CFA+PBS/ galectin-9

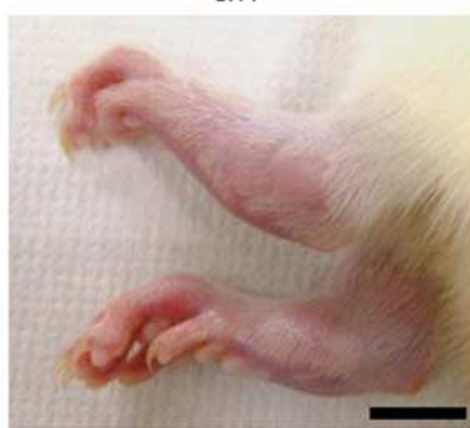

C

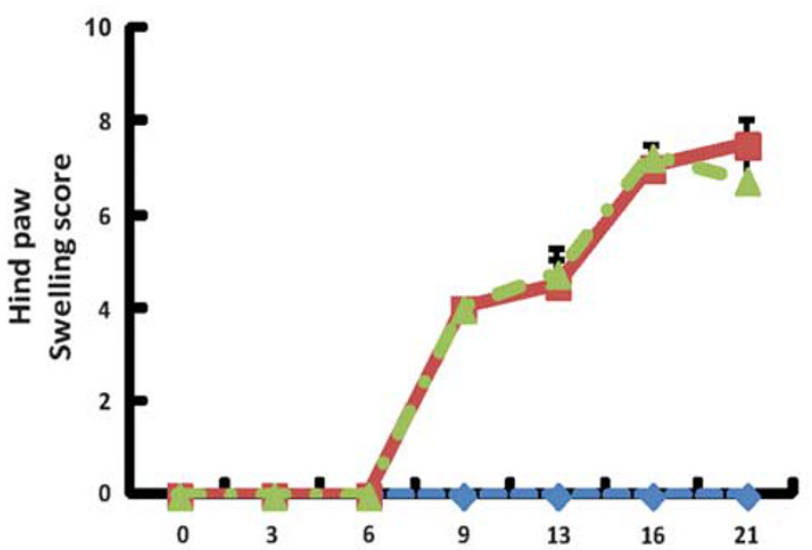

e

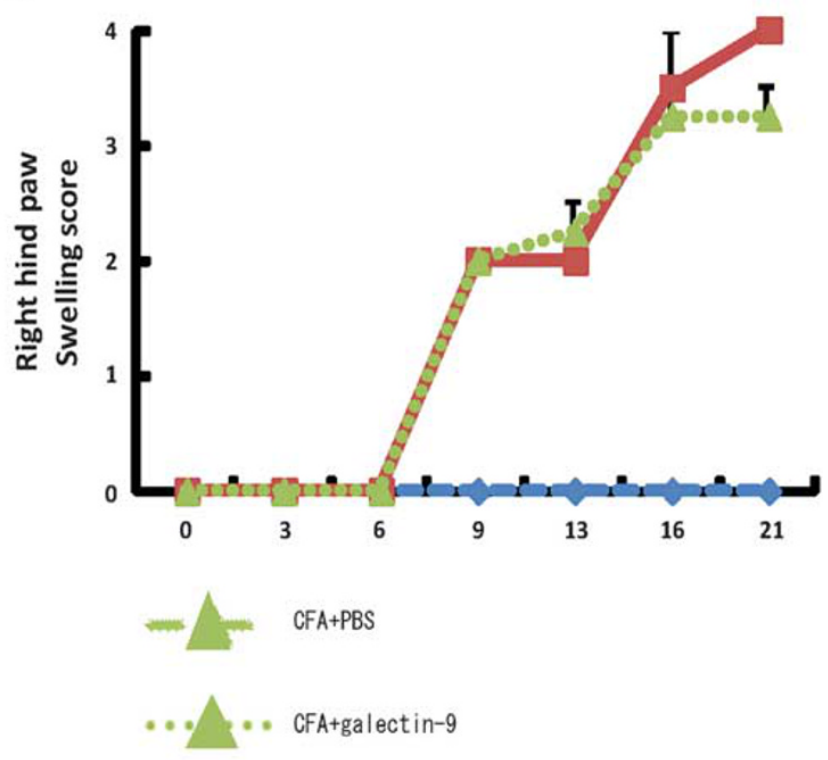

Figure 6 Effect on galectin-9 in the inflammatory parameters in arthritic rats. Galectin-9 $(5 \mu \mathrm{g})$ or vehicle control (phosphate-buffered saline (PBS)) was injected every 3 days into the area of ankle joints at the right ankle or the left ankle, respectively, in the same rat injected with complete Freund adjuvant (CFA) 2 days before. Every 3 or 4 days, arthritic scores were examined as described in Materials and methods. (a) Clinical observation of galectin-9-treated arthritic rats at 21 days after CFA injection. Time-course change in the arthritic score was shown as follows: sum of the swelling score of total 4 digits (swelling score) (b), swelling score of the right and left hind paw (c), and swelling score of the left hind paw injected only PBS

(d) and swelling score of the right hind paw injected galectin-9 (e). 
control

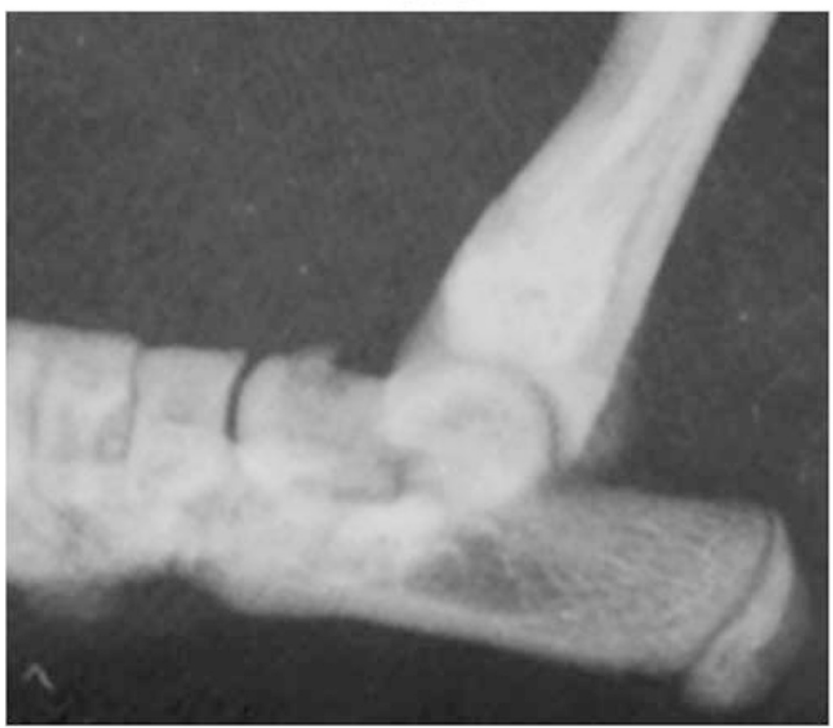

CFA/ PBS (Left ankle)

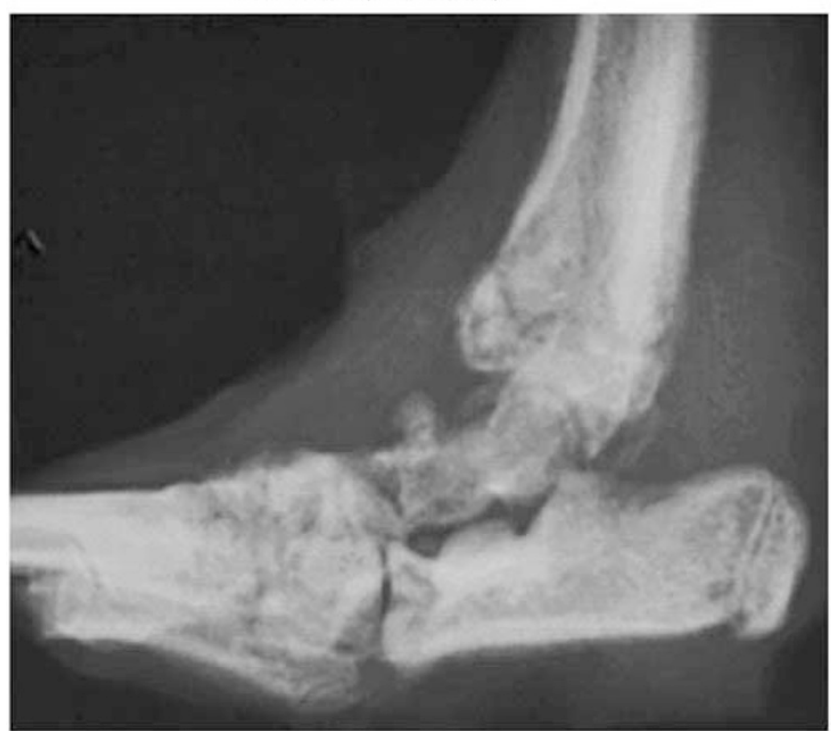

\section{CFA}

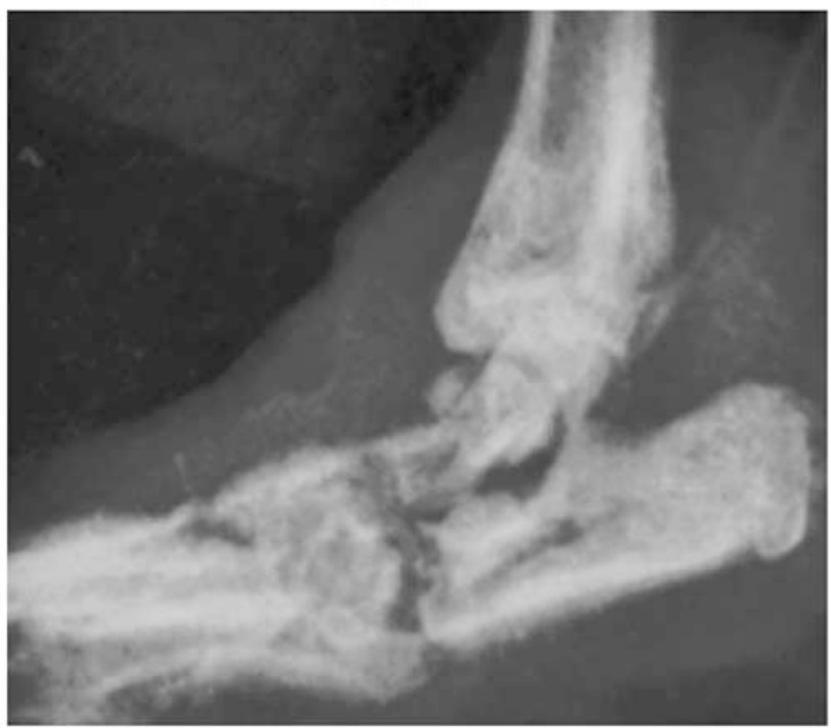

CFA/ galectin-9 (Right ankle)

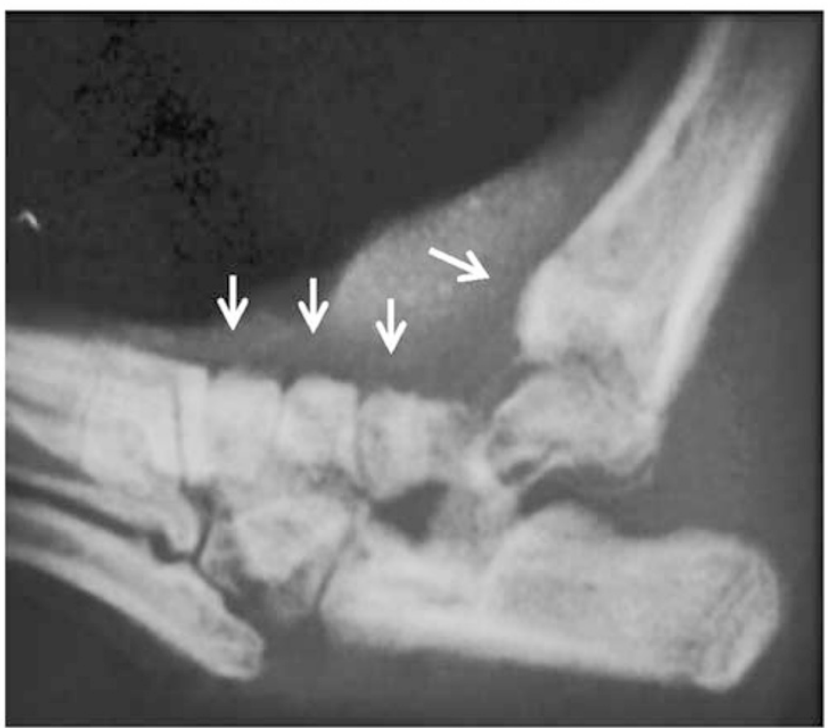

Figure 7 Marked suppression of bone destruction by the galectin- 9 administered into arthritic rats. Data show radiographic analysis of the ankle joints observed in Figure 6a. Arthritic rats were treated with phosphate-buffered saline (PBS) (left lower panel: complete Freund adjuvant CFA/PBS) or with galectin-9 (right lower panel: CFA/galectin-9). The injections were performed two times a week for 2 weeks beginning on day 2 after the adjuvant injection as described in the legends of Figure 6. At 21 days after CFA injection, hind paws were collected and analyzed by micro-CT as described in Materials and methods. Lower right panel (CFA/galectin-9) clearly shows the effect of galectin-9 on bone destruction in arthritic rats. The arrows indicate the portions of prevented bone destruction by the injection of galectin- 9 compared with CFA (upper right panel) and CFA/PBS (lower left panel). Data represent a typical experiments from three independent experiments.

that galectin-9 expression in the synovial membrane and synovial fluid was upregulated in patients with rheumatoid arthritis. ${ }^{26}$ However, it has been uncertain concerning an involvement of galectin-9 in the regulation of inflammatory bone destruction mediated by osteoclasts. Our present study strongly suggests an involvement of galectin-9 in the regulation of bone destruction in arthritic rats. Injected galectin-9 markedly suppressed bone destruction in arthritic rats without significant suppression of the inflammation. As galectin-9 is reported to suppress inflammation in arthritis, ${ }^{27}$ failure in the significant suppression of inflammation could be attributed to the low dose of galectin- 9 in our current experiments. However, the dose of galectin-9 injected to arthritic rats in our current experiments ( $5 \mu \mathrm{g}$ per injection) efficiently inhibited bone destruction, possibly through inhibiting osteoclastogenesis. Previously, we have reported on the suppression of bone destruction by galectin-3, in which galectin-3 efficiently suppressed bone destruction without affecting the level of inflammation in arthritic rats. ${ }^{22}$ Our current observations of the action of galectin- 9 on 
inflammatory bone destruction are similar to the action of galectin-3 in arthritic rats.

By immunohistochemical analysis, numerous Tim-3positive cells were observed over a wide area of the bone marrow cavity in the inflammatory bone destruction sites of arthritic rats. As Tim-3 is highly expressed on the surface of Th1 cells, Tim-3-positive cells observed in the bone marrow cavity of arthritic rats could be Th1 cells. However, Nakae et al. ${ }^{28}$ reported that Th17 cells also express Tim-3 in mice. Human Th17 cells also express Tim-3, although the expression level is much lower than in Th1 cells. ${ }^{16,29}$ Our observation of the numerous Tim-3-positive cells in the marrow cavity would reflect the presence of Th17 cells as well as Th1 cells in inflammatory bone destruction sites in arthritic rats. In our experiments, we have detected a high level of Tim-3 expression in the synovial membrane of arthritic rats, while no expression of this molecule was detected in the synovial membrane of normal rats, suggesting the presence of numerous helper T-cell populations (Th1 cell and Th17 cells) recruited to the synovial membrane with high level of inflammation in arthritic rats.

Our current findings on the selective expression of Tim- 3 in normal bone tissue was unexpected. Tim-3-positive area was almost restricted to the bone surface, which is different from the localization pattern of Tim-3-positive cells in arthritic rats, where Tim-3-positive cells were widely spread over the area of the bone marrow cavity. In normal rats, relatively large cells present on bone surface expressed Tim-3. Most cells of these Tim-3-positive cells were osteoclasts. We further obtained evidence showing the expression of Tim-3 mRNA and protein in osteoclast precursors and osteoclasts. As far as we know, this is the first report on the expression of Tim-3 in cells of osteoclast lineage. It has been reported that galectin-9 suppresses inflammation parameters in collagen-induced arthritis (CIA) in mice. ${ }^{26}$ Galectin-9 treatment was shown to downregulate proinflammatory cytokines (IL-17, IL-12, and IFN- $\gamma$ ) in joints in CIA mice. In our experiments, we started injection of galectin-9 2 days after the CFA injection in CIA in arthritic rats. To understand the therapeutic effect of galectin9 on bone destruction, galectin- 9 should be injected in the later stage when joint swelling has already been initiated. In our current study, injected galectin-9 markedly inhibited bone destruction almost without affecting the level of joint inflammation. It is probable that galectin-9 preferentially suppressed inflammatory bone destruction by inhibiting osteoclastogenesis rather than modulating inflammation in arthritic rats.

We investigated the immunohistochemical detection of endogenous galectin-9 in vivo. Galectin-9-positive cells were observed relatively in a limited area adjacent to bone in normal rats. In contrast, galectin-9-positive cells were present over the wide area of bone marrow cavity in the bone destruction site of arthritic rats. Localization pattern of galectin-9 in the distal tibia was similar to that of Tim-3, suggesting that the endogenous galectin-9 is acting on its receptor Tim-3 even in vivo.
Galectin-9 significantly suppressed osteoclastogenesis in vitro culture systems for evaluating osteoclastogenesis. Our previous report shows that suppression of osteoclastogenesis by galectin- 3 was not canceled by the addition of $\beta$-lactose. In contrast, galectin-9-mediated suppression of osteoclastogenesis was almost completely canceled by the addition of $\beta$-lactose. In these experiments, galactose, another antagonist of CRD, did not cancel the galectin-9-mediated suppression of osteoclastogenesis, suggesting that the monomeric sugar, galactose, is not sufficient to cancel interaction with Tim-3. Dimeric sugar, $\beta$-lactose, may be required to block the association of galectin-9 to Tim-3 on cell surface of osteoclast precursors. Our current data clearly shows that suppression of osteoclast differentiation by galectin- 9 was mediated through the CRD of galectin-9. By use of galectin-9 transgenic mice (Tg mice) and knockout mice, ${ }^{27}$ it has been demonstrated that the endogenous galectin-9 ameliorate CIA induced by the administration of the cocktail of anti-type II collagen monoclonal antibodies. In those experiments, clinical scores of arthritis were significantly reduced in Tg mice, whereas the clinical scores were markedly elevated in galectin-9 knockout mice. Galectin-9 has been reported to induce apoptosis of eosinophils, cancer cells, and T cells. Furthermore, galectin-9 preferentially induces apoptosis of CD4-positive $\mathrm{T}$ cells through the caspase-1 pathway. ${ }^{24,30}$ In our current study, galectin-9 significantly suppressed osteoclastogenesis without affecting the cell viability estimated by MTT assay. Therefore, it is unlikely that inhibition of osteoclastogenesis is attributed to the apoptotic activity of galectin-9 in cells in the osteoclast lineage.

Our study clearly shows that osteoclasts and osteoclast precursors expressed Tim-3 and the differentiation was markedly suppressed by its ligand galectin- 9 . These results suggest that galectin-9 suppresses osteoclastogenesis through Tim-3 expressed on cell surface of osteoclast precursors. Further studies using Tim-3 mutants ${ }^{31}$ will be required to reach a definitive conclusion concerning the signaling of Tim-3/galectin-9 system in osteoclast precursors. Seki et al. ${ }^{26}$ reported that galectin induces apoptosis of synovial fibroblasts obtained from patients with rheumatoid arthritis. Galectin-9 is known to induce apoptosis in CD4-positive T cells through the caspase-1 pathway. ${ }^{30}$ Although galectin- 9 markedly suppressed osteoclastogenesis without affecting cell viability, apoptotic events are not supposed to be involved to this suppression. Recently, Chiba et al. ${ }^{32}$ revealed a pivotal role of Tim-3 in the innate immunity in tumor microenvironments. In their findings, Tim-3 was elucidated to act as the receptor for the tumor cell-derived highmobility group box-1 (HMGB1), a strong inducer of endogenous innate immunity that is derived from necrotic tumor cells. ${ }^{33}$ In our current study, galectin-9 is supposed to be the ligand of Tim-3 expressed on cell surface of osteoclast precursors; however, HMGB1 could also act as the ligand for Tim-3 in inflammatory bone destruction site as the HMGB1 can also be derived from the activated dendritic cells and 
macrophages. It is known that rheumatoid arthritis is highly related to innate immunity, in which high level of toll-like receptors is expressed in macrophages and dendritic cells. ${ }^{34}$ Involvement of HMGB1 in the inflammatory bone destruction should also be explored. Abnormal augmentation in the activity of osteoclasts may be related to the marked increase in the innate immunity in rheumatoid arthritis.

Inflammatory bone destruction accompanying chronic inflammatory disease mediated by osteoclasts could be generally attenuated (negatively regulated) by the presence of the potential regulatory system Tim-3/galectin- 9 in the bone destruction sites in rheumatoid arthritis. It seems likely that inflammatory bone destruction mediated by active osteoclasts could be efficiently controlled by the administration of recombinant galectin-9 in rheumatoid arthritis. Abrogating the Tim-3/galectin-9 system could lead to the incidence of some vicious cycle of augment in the inflammation itself and inflammatory bone destruction.

Supplementary Information accompanies the paper on the Laboratory Investigation website (http://www.laboratoryinvestigation.org)

\section{ACKNOWLEDGMENTS}

We thank Dr Yasuyoshi Osaki, Dr Takayoshi Yamaza, Dr Tomoko Kitsuki and Dr Mizuho Kido of Kyushu University, Faculty of Dental Science for helpful suggestions. This work is funded in part by a Grant-in-Aid for Scientific Research (B) and (Challenging Exploratory Research) of Japan Society for Promotion of Science (JSPS) (project nos. 21390492, 21659424, 23659860, 25670780, and 26670803).

\section{DISCLOSURE/CONFLICT OF INTEREST}

The authors declare no conflict of interest.

1. Vasta GR. Roles of galectins in infection. Nat Rev Microbiol 2009;7:424-438.

2. Grigorian A, Torossian S, Demetriou M. T-cell growth, cell surface organization, and the galectin-glycoprotein lattice. Immunol Rev 2009;230:232-246.

3. Wada J, Kanwar YS. Identification and characterization of galectin-9, a novel beta-galactoside-binding mammalian lectin. J Biol Chem 1997;272:6078-6086.

4. Matsumoto $\mathrm{R}$, Matsumoto $\mathrm{H}$, Seki $\mathrm{M}$, et al. Human ecalectin, a variant of human galectin-9, is a novel eosinophil chemoattractant produced by T lymphocytes. J Biol Chem 1998;273:16976-16984.

5. Tsuboi $Y$, Abe H, Nakagawa R, et al. Galectin-9 protects mice from the Shwartzman reaction by attracting prostaglandin E2-producing polymorphonuclear leukocytes. Clin Immunol 2007;124:221-233.

6. Nobumoto A, Oomizu S, Arikawa T, et al. Galectin-9 expands unique macrophages exhibiting plasmacytoid dendritic cell-like phenotypes that activate NK cells in tumor-bearing mice. Clin Immunol 2009; 130:322-330.

7. McMorrow JP, Murphy EP. Inflammation: a role for NR4A orphan nuclear receptors? Biochem Soc Trans 2011;39:688-693.

8. Sakai $H$, Jingushi $S$, Shuto $T$, et al. Fibroblasts from the inner granulation tissue of the pseudocapsule in hips at revision arthroplasty induce osteoclast differentiation, as do stromal cells. Ann Rheum Dis 2002;61:103-109.

9. Gaffen $\mathrm{SL}$, Hajishengallis $\mathrm{G}$. A new inflammatory cytokine on the block: re-thinking periodontal disease and the Th1/Th2 paradigm in the context of Th17 cells and IL-17. J Dent Res 2008;87:817-828.
10. Sakuishi K, Jayaraman P, Behar SM, et al. Emerging Tim-3 functions in antimicrobial and tumor immunity. Trends Immunol 2011;32:345-349.

11. Rangachari M, Kuchroo VK. Using EAE to better understand principles of immune function and autoimmune pathology. J Autoimmun 2013;45:31-39.

12. Monney L, Sabatos CA, Gaglia JL, et al. Th1-specific cell surface protein Tim-3 regulates macrophage activation and severity of an autoimmune disease. Nature 2002;415:536-541.

13. Bettelli $E$, Korn $T$, Oukka $M$, et al. Induction and effector functions of $\mathrm{T}(\mathrm{H}) 17$ cells. Nature 2008;453:1051-1057.

14. Ariel $A$, Timor $O$. Hanging in the balance: endogenous antiinflammatory mechanisms in tissue repair and fibrosis. J Pathol 2013;229:250-263.

15. Jayaraman P, Sada-Ovalle I, Beladi S, et al. Tim3 binding to galectin-9 stimulates antimicrobial immunity. J Exp Med 2010;207:2343-2354.

16. Hastings WD, Anderson DE, Kassam N, et al. TIM-3 is expressed on activated human CD4 + T cells and regulates Th1 and Th17 cytokines. Eur J Immunol 2009;39:2492-2501.

17. Kukita A, Kukita T, Nagata K, et al. The transcription factor FBI-1/OCZF/ LRF is expressed in osteoclasts and regulates RANKL-induced osteoclast formation in vitro and in vivo. Arthritis Rheum 2011;63: 2744-2754.

18. Kukita A, Kukita T, Hata K, et al. Heat-treated osteoblastic cell (ROS17/ 2.8)-conditioned medium induces the formation of osteoclast-like cells. Bone Miner 1993;23:113-127.

19. Kukita $A$, Kukita $T$, Shin $J \mathrm{H}$, et al. Induction of mononuclear precursor cells with osteoclastic phenotypes in a rat bone marrow culture system depleted of stromal cells. Biochem Biophys Res Comm 1993; 196:1383-1389.

20. Watanabe T, Kukita T, Kukita A, et al. Direct stimulation of osteoclastogenesis by MIP-1alpha: evidence obtained from studies using RAW264 cell clone highly responsive to RANKL. J Endocrinol 2004;180:193-201.

21. Kukita T, Wada N, Kukita A, et al. RANKL-induced DC-STAMP is essential for osteoclastogenesis. J Exp Med 2004;200:941-946.

22. Li YJ, Kukita A, Teramachi J, et al. A possible suppressive role of galectin-3 in upregulated osteoclastogenesis accompanying adjuvantinduced arthritis in rats. Lab Invest 2009;89:26-37.

23. Li YJ, Kukita A, Watanabe T, et al. Nordihydroguaiaretic acid inhibition of NFATC1 suppresses osteoclastogenesis and arthritis bone destruction in rats. Lab Invest 2012;92:1777-1787.

24. Seki M, Oomizu S, Sakata KM, et al. Galectin-9 suppresses the generation of Th17, promotes the induction of regulatory $T$ cells, and regulates experimental autoimmune arthritis. Clin Immunol 2008; 127:78-88.

25. Bottini N, Firestein GS. Duality of fibroblast-like synoviocytes in RA: passive responders and imprinted aggressors. Nat Rev Rheumatol 2013;9:24-33.

26. Seki M, Sakata KM, Oomizu S, et al. Beneficial effect of galectin 9 on rheumatoid arthritis by induction of apoptosis of synovial fibroblasts. Arthritis Rheum 2007;56:3968-3976.

27. Arikawa T, Watanabe K, Seki M, et al. Galectin-9 ameliorates immune complex-induced arthritis by regulating Fc gamma $\mathrm{R}$ expression on macrophages. Clin Immunol 2009;133:382-392.

28. Nakae S, Iwakura $\mathrm{Y}$, Suto $\mathrm{H}$, et al. Phenotypic differences between Th1 and Th17 cells and negative regulation of Th1 cell differentiation by IL17. J Leuk Biol 2007;81:1258-1268.

29. Kanai $Y$, Satoh T, Igawa K, et al. Impaired expression of Tim-3 on Th17 and Th1 cells in psoriasis. Acta Derm Venereol 2012;92:367-371.

30. Kashio Y, Nakamura K, Abedin MJ, et al. Galectin-9 induces apoptosis through the calcium-calpain-caspase-1 pathway. J Immunol 2003;170: 3631-3636.

31. Lee J, Su EW, Zhu C, et al. Phosphotyrosine-dependent coupling of Tim-3 to T-cell receptor signaling pathways. Mol Cell Biol 2011;31: 3963-3974.

32. Chiba S, Baghdadi M, Akiba $\mathrm{H}$, et al. Tumor-infiltrating DCs suppress nucleic acid-mediated innate immune responses through interactions between the receptor TIM-3 and the alarmin HMGB1. Nat Immunol 2012;13:832-842.

33. Tang D, Lotze MT. Tumor immunity times out: TIM-3 and HMGB1. Nat Immunol 2012;13:808-810.

34. Goh FG, Midwood KS. Intrinsic danger: activation of Toll-like receptors in rheumatoid arthritis. Rheumatology 2012;51:7-23. 